\title{
The Average Errors for Linear Combinations of Bernstein Operators on the Wiener Space*
}

\author{
Yanjie Jiang", Ziqing Zhang \\ Department of Mathematics and Physics, North China Electric Power University, Baoding, China \\ Email: ${ }^{\text {jiangyj@126.com }}$
}

Received September 18, 2013; revised October 20, 2013; accepted November 4, 2013

Copyright (c) 2013 Yanjie Jiang, Ziqing Zhang. This is an open access article distributed under the Creative Commons Attribution License, which permits unrestricted use, distribution, and reproduction in any medium, provided the original work is properly cited.

\begin{abstract}
In this paper, we discuss the average errors of function approximation by linear combinations of Bernstein operators. The strongly asymptotic orders for the average errors of the combinations of Bernstein operators sequence are determined on the Wiener space.
\end{abstract}

Keywords: Linear Combinations; Bernstein Operators; Weighted $L_{p}$-Norm; Average Error; Wiener Space

\section{Introduction}

Let $F$ be a real separable Banach space equipped with a probability measure $\mu$ on the Borel sets of $F$. Let $X$ be another normed space such that $F$ is continuously embedded in $X$. By $\|\cdot\|_{X}$ we denote the norm in $X$. Any $T: F \rightarrow X$ such that $f \mapsto\|f-T(f)\|_{X}$ is a measurable mapping is called an approximation operator. The $p$-average error of $T$ is defined as

$$
e_{p}\left(T, F,\|\cdot\|_{X}, \mu\right):=\left(\int\|f-T(f)\|_{X}^{p} \mu(\mathrm{d} f)\right)^{1 / p} .
$$

Let

$$
F_{0}:=\{f \in C[0,1] \mid f(0)=0\} .
$$

For every $f \in F_{0}$ set

$$
\|f\|_{C}:=\max _{0 \leq t \leq 1}|f(t)| .
$$

Then $\left(F_{0},\|\cdot\|_{C}\right)$ becomes a separable Banach space. Denote by $\mathfrak{B}\left(F_{0}\right)$ the Borel class of $\left(F_{0},\|\cdot\|_{C}\right)$ and by $\omega_{0}$ the Wiener measure on $\mathfrak{B}\left(F_{0}\right)$ (see [1]). From [1, p. 70] we know

$$
\begin{aligned}
& \int_{F_{0}} f(s) f(t) \omega_{0}(\mathrm{~d} f) \\
& =\min \{s, t\}=\frac{1}{2}(s+t-|s-t|), \quad \forall s, t \in[0,1] .
\end{aligned}
$$

\footnotetext{
"Supported by National Natural Science Foundation of China (Project no 10871132 and 11271263) and by a grant from Hebei province higher school science and technology research (Z2010160).

"Corresponding author.
}

The Bernstein operator on $C[0,1]$ defined by

$$
B_{n}(f, x):=\sum_{k=0}^{n} f\left(\frac{k}{n}\right) p_{n, k}(x),
$$

where

$$
p_{n, k}(x)=\left(\begin{array}{l}
n \\
k
\end{array}\right) x^{k}(1-x)^{n-k}, k=0,1, \cdots, n .
$$

This operator turned out to be a very interesting operator, easy to deal with and having many applications in approximation theory and practice.

Since Bernstein operators cannot be used in the investigation of higher orders of smoothness, Butzer [2] introduced combinations of Bernstein operators. Ditzian and Totik [3, p. 116] extended this method and defined the combinations as

$$
L_{n, m}(f, x):=\sum_{i=0}^{m-1} C_{i}(n) B_{n_{i}}(f, x),
$$

where $n_{i}$ and $C_{i}(n)$ satisfy the following conditions:
(a) $n=n_{0}<\cdots<n_{m-1} \leq C n$;
(b) $\sum_{i=0}^{m-1}\left|C_{i}(n)\right| \leq C$;
(c) $\sum_{i=0}^{m-1} C_{i}(n)=1$;
(d) $\sum_{i=0}^{m-1} C_{i}(n) n_{i}^{-\rho}=0, \quad \rho=1,2, \cdots, m-1$.

Throughout this paper, $C$ denotes a positive constant 
independent of $n$ and $x$, which may be a different constant in different cases.

For

$$
\rho \in L_{1}[0,1], \rho \geq 0,1 \leq p<\infty,
$$

the weighted $L_{p}$-norm of $f \in C[0,1]$ is defined by

$$
\|f\|_{p, \rho}:=\left(\int_{0}^{1}|f(t)|^{p} \cdot \rho(t) \mathrm{d} t\right)^{\frac{1}{p}} .
$$

\section{Main Result}

Recently G. Q. Xu [4] studied the average errors of Bernstein operators approximation on the Wiener space. Motivated by [4], we considered the average errors of function approximation by linear combinations of Bernstein operators. The strongly asymptotic orders for the average errors of the linear combinations of Bernstein operators sequence are determined on the Wiener space. We obtain:

Theorem 1. Let $1 \leq p<\infty, L_{n, m}(f, x)$ be given by (2), $\rho \in L_{1}[0,1], \rho(x)>0$ and $\rho(x)$ is continuous on $(0,1)$. Then we have

$$
\begin{aligned}
e_{p}\left(L_{n, m}, F_{0},\|\cdot\|_{p, \rho}, \omega_{0}\right) & {\left[\sum_{i=0}^{m-1} \sum_{j=0}^{m-1} C_{i}(n) C_{j}(n)\left(\sqrt{\frac{2}{\pi n_{i}}}-\frac{\sqrt{\frac{n_{i}}{n_{j}}}+\sqrt{\frac{n_{j}}{n_{i}}}}{\sqrt{2 \pi\left(n_{i}+n_{j}\right)}}\right)\right]^{\frac{1}{2}} } \\
& \cdot\left(v_{p} \int_{0}^{1}(x(1-x))^{\frac{p}{4}} \rho(x) \mathrm{d} x\right)^{\frac{1}{p}}+\circ\left(n^{-\frac{1}{4}}\right),
\end{aligned}
$$

where

$$
v_{p}=\frac{1}{\sqrt{2 \pi}} \int_{-\infty}^{+\infty}|x|^{p} \mathrm{e}^{-\frac{x^{2}}{2}} \mathrm{~d} x .
$$

Here and in the following the notation $a_{n}=\circ\left(b_{n}\right)$ for sequences $\left\{a_{n}\right\}$ and $\left\{b_{n}\right\}$ means that

$$
\lim _{n \rightarrow \infty} a_{n} / b_{n}=0 \text {. }
$$

\section{Proof of Theorem 1}

To prove Theorem 1 we need the following two lemmas.

Lemma 1([5, p. 15]). If

$$
0<\alpha<\frac{1}{2},
$$

then

$$
\sum_{\left|\frac{k}{n}-x\right| \geq n^{-\alpha}} p_{n, k}(x) \leq C n^{-k}
$$

for each $k>0$, the constant $C$ depending only on $\alpha$ and $k$.

Lemma 2 ([5, p. 15]). For fixed

$$
0<x<1, \delta>\frac{1}{3}
$$

the asymptotic relation

$$
\begin{aligned}
& p_{n, k}(x)=\left(\begin{array}{l}
n \\
k
\end{array}\right) x^{k}(1-x)^{n-k} \\
& \cong(2 \pi x(1-x) n)^{-\frac{1}{2}} \exp \left\{-\frac{n}{2 x(1-x)}\left(\frac{k}{n}-x\right)^{2}\right\} \\
& =P_{n, k}(x)
\end{aligned}
$$

holds uniformly for all values of $k$ satisfying the inequality

$$
\left|\frac{k}{n}-x\right| \leq n^{-\delta}
$$

In other words,

$$
\lim _{n \rightarrow \infty} p_{n, k}(x) / P_{n, k}(x)=1
$$

uniformly for all $k$ satisfying (4).

Proof of Theorem 1. From [1, p.107] we have

$$
\begin{aligned}
& e_{p}^{p}\left(L_{n, m}, F_{0},\|\cdot\|_{p, \rho}, \omega_{0}\right) \\
& =v_{p} \int_{0}^{1}\left(\int_{F_{0}}\left|f(x)-L_{n, m}(f, x)\right|^{2} \omega_{0}(\mathrm{~d} f)\right)^{\frac{p}{2}} \rho(x) \mathrm{d} x .
\end{aligned}
$$

By (2),

$$
\begin{aligned}
& \int_{F_{0}}\left|f(x)-L_{n, m}(f, x)\right|^{2} \omega_{0}(\mathrm{~d} f) \\
& =\int_{F_{0}} f^{2}(x) \omega_{0}(\mathrm{~d} f)-2 \sum_{i=0}^{m-1} C_{i}(n) \sum_{k=0}^{n_{i}} p_{n_{i}, k}(x) \\
& \quad \cdot \int_{F_{0}} f(x) f\left(\frac{k}{n_{i}}\right) \omega_{0}(\mathrm{~d} f)+\sum_{i=0}^{m-1} C_{i}(n) \sum_{j=0}^{m-1} C_{j}(n) \\
& \quad \cdot \sum_{k=0}^{n_{i}} p_{n_{i}, k}(x) \sum_{s=0}^{n_{j}} p_{n_{j}, s}(x) \int_{F_{0}} f\left(\frac{k}{n_{i}}\right) f\left(\frac{s}{n_{j}}\right) \omega_{0}(\mathrm{~d} f) \\
& =A_{1}(x)-2 A_{2}(x)+A_{3}(x) .
\end{aligned}
$$

On using (1), we obtain

$$
A_{1}(x)=\int_{F_{0}} f^{2}(x) \omega_{0}(\mathrm{~d} f)=x .
$$

Note that

$$
\begin{aligned}
& \sum_{k=0}^{n} p_{n, k}(x)=1, \quad \sum_{k=0}^{n} k p_{n, k}(x)=n x, \\
& \sum_{k=0}^{n} k^{2} p_{n, k}(x)=n^{2} x^{2}+n x(1-x),
\end{aligned}
$$




$$
\begin{aligned}
A_{2}(x) & =\sum_{i=0}^{m-1} C_{i}(n) \sum_{k=0}^{n_{i}} p_{n_{i}, k}(x) \int_{F_{0}} f(x) f\left(\frac{k}{n_{i}}\right) \omega_{0}(\mathrm{~d} f) \\
& =\sum_{i=0}^{m-1} C_{i}(n) \sum_{k=0}^{n_{i}} p_{n_{i}, k}(x) \frac{1}{2}\left(x+\frac{k}{n_{i}}-\left|x-\frac{k}{n_{i}}\right|\right) \\
& =x-\frac{1}{2} \sum_{i=0}^{m-1} C_{i}(n) \sum_{k=0}^{n_{i}} p_{n_{i}, k}(x)\left|x-\frac{k}{n_{i}}\right|
\end{aligned}
$$

From [4,(3.24)], we know

$$
\sum_{k=0}^{n_{i}} p_{n_{i}, k}(x)\left|x-\frac{k}{n_{i}}\right|=\frac{\sqrt{2 x(1-x)}}{\sqrt{\pi n_{i}}}+\circ\left(n_{i}^{-\frac{1}{2}}\right) .
$$

Combining(3) and (9) we get

$$
\begin{aligned}
A_{2}(x) & =x-\frac{1}{2} \sum_{i=0}^{m-1} C_{i}(n)\left(\frac{\sqrt{2 x(1-x)}}{\sqrt{\pi n_{i}}}+\circ\left(n_{i}^{-\frac{1}{2}}\right)\right) \\
& =x-\sqrt{\frac{x(1-x)}{2 \pi}} \sum_{i=0}^{m-1} \frac{C_{i}(n)}{\sqrt{n_{i}}}+\circ\left(n^{-\frac{1}{2}}\right) .
\end{aligned}
$$

Now, we estimate the term $A_{3}(x)$. From (2) and (8),

$$
\begin{aligned}
A_{3}(x)= & \sum_{i=0}^{m-1} C_{i}(n) \sum_{j=0}^{m-1} C_{j}(n) \sum_{k=0}^{n_{i}} p_{n_{i}, k}(x) \sum_{s=0}^{n_{j}} p_{n_{j}, s}(x) \\
& \quad \int_{F_{0}} f\left(\frac{k}{n_{i}}\right) f\left(\frac{s}{n_{j}}\right) \omega_{0}(\mathrm{~d} f) \\
= & \sum_{i=0}^{m-1} C_{i}(n) \sum_{j=0}^{m-1} C_{j}(n) \sum_{k=0}^{n_{i}} p_{n_{i}, k}(x) \sum_{s=0}^{n_{j}} p_{n_{j}, s}(x) \\
& \cdot \frac{1}{2}\left(\frac{k}{n_{i}}+\frac{s}{n_{j}}-\left|\frac{k}{n_{i}}-\frac{s}{n_{j}}\right|\right) \\
= & x-\frac{1}{2} \sum_{i=0}^{m-1} C_{i}(n) \sum_{j=0}^{m-1} C_{j}(n) \sum_{k=0}^{n_{i}} p_{n_{i}, k}(x) \\
& \cdot \sum_{s=0}^{n_{j}} p_{n_{j}, s}(x)\left|\frac{k}{n_{i}}-\frac{s}{n_{j}}\right| .
\end{aligned}
$$

$$
\begin{aligned}
= & \sum_{k=0}^{n_{i}} p_{n_{i}, k}(x) \sum_{\left|\frac{s}{n_{j}}-x\right|>n_{j}^{-5 / 12}} p_{n_{j}, s}(x)\left|\frac{k}{n_{i}}-\frac{s}{n_{j}}\right| \\
& +\sum_{\left|\frac{k}{n_{i}}-x\right|>n_{i}^{-5 / 12}} p_{n_{i}, k}(x) \sum_{\left|\frac{s}{n_{j}}-x\right| \leq n_{j}^{-5 / 12}} p_{n_{j}, s}(x)\left|\frac{k}{n_{i}}-\frac{s}{n_{j}}\right| \\
& +\sum_{\left|\frac{k}{n_{i}}-x\right| \leq n_{i}^{-5 / 12}} p_{n_{n_{i}}, k}(x) \sum_{\left|\frac{s}{n_{j}}-x\right| \leq n_{j}^{-5 / 12}} p_{n_{j}, s}(x)\left|\frac{k}{n_{i}}-\frac{s}{n_{j}}\right| \\
= & 0\left(n^{-\frac{23}{12}}\right)+\sum_{\left|\frac{k}{n_{i}}-x\right| \leq n_{i}^{-5 / 12}} p_{n_{i}, k}(x) \sum_{\left|\frac{s}{n_{j}}-x\right| \leq n_{j}^{-5 / 12}} p_{n_{j}, s}(x)\left|\frac{k}{n_{i}}-\frac{s}{n_{j}}\right| .
\end{aligned}
$$

For

$$
\left|\frac{k}{n_{i}}-x\right| \leq n_{i}^{-5 / 12} \text { and }\left|\frac{s}{n_{j}}-x\right| \leq n_{j}^{-5 / 12},
$$

by Lemma 2,

$$
\begin{aligned}
\left|\frac{k}{n_{i}}-\frac{s}{n_{j}}\right| p_{n_{i}, k}(x) p_{n_{j}, s}(x) \\
=\frac{1+o(1)}{2 \pi x(1-x) \sqrt{n_{i} n_{j}}}\left|\frac{k}{n_{i}}-\frac{s}{n_{j}}\right| \\
\quad \cdot \exp \left\{\frac{-n_{i}}{2 x(1-x)}\left(x-\frac{k}{n_{i}}\right)^{2}+\frac{-n_{j}}{2 x(1-x)}\left(x-\frac{s}{n_{j}}\right)^{2}\right\} .
\end{aligned}
$$

Set

$$
F\left(u_{1}, u_{2}\right):=\left(u_{1}-u_{2}\right) \exp \left\{\frac{-n_{i}}{2 x(1-x)} u_{1}^{2}+\frac{-n_{j}}{2 x(1-x)} u_{2}^{2}\right\} .
$$

For

$$
\left(v_{1}, v_{2}\right) \in\left[\frac{k-1}{n_{i}}, \frac{k+1}{n_{i}}\right] \times\left[\frac{s-1}{n_{j}}, \frac{s+1}{n_{j}}\right]
$$

by the differential mean value theorem we have

$$
\sum_{\frac{s}{n_{j}}-x \mid>n_{j}^{-5 / 12}} p_{n_{j}, s}(x) \leq C n^{-2} \text {. }
$$

Note that

$$
\begin{gathered}
0 \leq \frac{k}{n_{i}}, \frac{s}{n_{j}} \leq 1, \\
\sum_{\mid \frac{s}{n_{j}}-x>n_{j}^{-5 / 22}} p_{n_{j}, s}(x)\left|\frac{k}{n_{i}}-\frac{s}{n_{j}}\right| \leq C n^{-2} .
\end{gathered}
$$

we get

By (8) and (12), we obtain

$$
\begin{aligned}
& \left(\left(x-\frac{k}{n_{i}}\right)-\left(x-\frac{s}{n_{j}}\right)\right) \exp \left\{\frac{-n_{i}}{2 x(1-x)}\left(x-\frac{k}{n_{i}}\right)^{2}\right. \\
& \left.+\frac{-n_{j}}{2 x(1-x)}\left(x-\frac{s}{n_{j}}\right)^{2}\right\}-\left(\left(x-v_{1}\right)-\left(x-v_{2}\right)\right) \\
& \cdot \exp \left\{\frac{-n_{i}}{2 x(1-x)}\left(x-v_{1}\right)^{2}+\frac{-n_{j}}{2 x(1-x)}\left(x-v_{2}\right)^{2}\right\} \\
& =F_{1}^{\prime}(\xi, \eta)\left(v_{1}-\frac{k}{n_{i}}\right)+F_{2}^{\prime}(\xi, \eta)\left(v_{2}-\frac{s}{n_{j}}\right),
\end{aligned}
$$


where

$$
\begin{aligned}
(\xi, \eta) \in\left(x-\frac{k+1}{n_{i}}, x-\frac{k-1}{n_{i}}\right) \times\left(x-\frac{s+1}{n_{j}}, x-\frac{s-1}{n_{j}}\right) & \quad\left|F_{1}^{\prime}(\xi, \eta)\right| \leq C, \quad\left|F_{2}^{\prime}(\xi, \eta)\right| \leq C . \\
& \left|\frac{k}{n_{i}}-\frac{s}{n_{j}}\right| \exp \left\{\frac{-n_{i}}{2 x(1-x)}\left(x-\frac{k}{n_{i}}\right)^{2}+\frac{-n_{j}}{2 x(1-x)}\left(x-\frac{s}{n_{j}}\right)^{2}\right\} \\
& =\left|\left(x-v_{1}\right)-\left(x-v_{2}\right)\right| \exp \left\{\frac{-n_{i}}{2 x(1-x)}\left(x-v_{1}\right)^{2}+\frac{-n_{j}}{2 x(1-x)}\left(x-v_{2}\right)^{2}\right\}+\left(n^{-\frac{11}{12}}\right) .
\end{aligned}
$$

Integrating two side of (16) about $\left(v_{1}, v_{2}\right)$ in

$$
\left[\frac{k}{n_{i}}, \frac{k+1}{n_{i}}\right] \times\left[\frac{s}{n_{j}}, \frac{s+1}{n_{j}}\right]
$$

we get

$$
\begin{aligned}
& \left|\frac{k}{n_{i}}-\frac{s}{n_{j}}\right| \exp \left\{\frac{-n_{i}}{2 x(1-x)}\left(x-\frac{k}{n_{i}}\right)^{2}+\frac{-n_{j}}{2 x(1-x)}\left(x-\frac{s}{n_{j}}\right)^{2}\right\} \\
& =n_{i} n_{j} \int_{\frac{k}{n_{i}}}^{\frac{k+1}{n_{i}}} \mathrm{~d} v_{1} \int_{\frac{s}{n_{j}}}^{\frac{s+1}{n_{j}}}\left|\left(x-v_{1}\right)-\left(x-v_{2}\right)\right| \cdot \exp \left\{\frac{-n_{i}}{2 x(1-x)} \cdot\left(x-v_{1}\right)^{2}+\frac{-n_{j}}{2 x(1-x)}\left(x-v_{2}\right)^{2}\right\} \mathrm{d} v_{2}+\left(n^{-\frac{11}{12}}\right) .
\end{aligned}
$$

From (14)-(17), we have

$$
\begin{aligned}
& \sum_{\left|\frac{k}{n_{i}}-x\right| \leq n_{i}^{-5 / 12}} p_{n_{i}, k}(x) \sum_{\left|\frac{s}{n_{j}}-x\right| \leq n_{j}^{-5 / 12}} p_{n_{j}, s}(x)\left|\frac{k}{n_{i}}-\frac{s}{n_{j}}\right| \\
= & \sum_{\left|\frac{k}{n_{i}}-x\right| \leq n_{i}^{-5 / 12}} \sum_{\left|\frac{s}{n_{j}}-x\right| \leq n_{j}^{-5 / 12} \mid}\left|\frac{k}{n_{i}}-\frac{s}{n_{j}}\right| p_{n_{i}, k}(x) p_{n_{j}, s}(x) \\
= & \sum_{\left|\frac{k}{n_{i}}-x\right| \leq n_{i}^{-5 / 12}} \sum_{\frac{s}{n_{j}}-x \mid \leq n_{j}^{-5 / 12}} \frac{(1+\circ(1)) \sqrt{n_{i} n_{j}}}{2 \pi x(1-x)} \int_{\frac{k}{n_{i}}}^{\frac{k+1}{n_{i}}} \mathrm{~d} v_{1} \int_{\frac{s}{n_{j}}}^{\frac{s+1}{n_{j}}} \\
& \left(x-v_{1}\right)-\left(x-v_{2}\right) \mid \cdot \exp \left\{\frac{-n_{i}}{2 x(1-x)}\left(x-v_{1}\right)^{2}+\frac{-n_{j}}{2 x(1-x)}\left(x-v_{2}\right)^{2}\right\} \mathrm{d} v_{2}+\circ\left(n^{-\frac{23}{12}}\right) \\
= & \sum_{\left|\frac{k}{n_{i}}-x\right| \leq n_{i}^{-5 / 12}} \sum_{\frac{s}{n_{j}}-x \mid \leq n_{j}^{-5 / 12}} \frac{\sqrt{n_{i} n_{j}}}{2 \pi x(1-x)} \int_{\frac{k}{n_{i}}}^{\frac{k+1}{n_{i}}} \mathrm{~d} v_{1} \int_{\frac{s}{n_{j}}}^{\frac{s+1}{n_{j}}}\left|\left(x-v_{1}\right)-\left(x-v_{2}\right)\right| \exp \left\{\frac{-n_{i}}{2 x(1-x)}\left(x-v_{1}\right)^{2}+\frac{-n_{j}}{2 x(1-x)}\left(x-v_{2}\right)^{2}\right\} \mathrm{d} v_{2} \\
& +\circ\left(n^{-1}\right) \\
= & \frac{\sqrt{n_{i} n_{j}}}{2 \pi x(1-x)} \int_{x-n_{i}^{-5 / 12}}^{x+n_{i}^{-5 / 12}+\frac{1}{n_{i}}} d v_{1} \int_{x-n_{j}}^{x+n_{j}^{-5 / 12}}+\frac{1}{n_{j}}\left|\left(x-v_{1}\right)-\left(x-v_{2}\right)\right| \exp \left\{\frac{-n_{i}}{2 x(1-x)}\left(x-v_{1}\right)^{2}+\frac{-n_{j}}{2 x(1-x)}\left(x-v_{2}\right)^{2}\right\} \mathrm{d} v_{2}+\circ\left(n^{-1}\right) .
\end{aligned}
$$

Let

$$
w_{1}=\sqrt{\frac{n_{i}}{2 x(1-x)}}\left(x-v_{1}\right), w_{2}=\sqrt{\frac{n_{j}}{2 x(1-x)}}\left(x-v_{2}\right) \text {, }
$$

by (18), we get 


$$
\begin{aligned}
& \sum_{\left|\frac{k}{n_{i}}-x\right| \leq n_{i}^{-5 / 12}} p_{n_{i}, k}(x) \sum_{\left|\frac{s}{n_{j}}-x\right| \leq n_{j}^{-5 / 12}} p_{n_{j}, s}(x)\left|\frac{k}{n_{i}}-\frac{s}{n_{j}}\right| \\
& =\frac{\sqrt{2 x(1-x)}}{\pi} \int_{\frac{-n_{i}^{1 / 12}-n_{i}^{-1 / 2}}{\sqrt{2 x(1-x)}}}^{\frac{n_{i}^{1 / 12}}{\sqrt{2(1-x)}}} \mathrm{d} w_{1} \int_{-\frac{n_{j}^{1 / 12}-n_{j}^{-j / 2}}{\sqrt{2 x(1-x)}}}^{\frac{n_{j}^{1 / 12}}{\sqrt{2 x(1-x}}}\left|\frac{w_{1}}{\sqrt{n_{i}}}-\frac{w_{2}}{\sqrt{n_{j}}}\right| \cdot \exp \left\{-w_{1}^{2}-w_{2}^{2}\right\} \mathrm{d} w_{2}+\circ\left(n^{-1}\right) .
\end{aligned}
$$

By (3), suppose that

$$
\frac{n}{n_{i}}=c_{i}^{2}, \frac{n}{n_{j}}=c_{j}^{2},
$$

from (19) and the convergence of the improper integral

$$
\int_{-\infty}^{+\infty} \mathrm{d} w_{1} \int_{-\infty}^{+\infty}\left|c_{i} w_{1}-c_{j} w_{2}\right| \exp \left\{-w_{1}^{2}-w_{2}^{2}\right\} \mathrm{d} w_{2},
$$

we have

$$
\begin{aligned}
& \sum_{\left|\frac{k}{n_{i}}-x\right| \leq n_{i}^{-5 / 12}} p_{n_{i}, k}(x) \sum_{\left|\frac{s}{n_{j}}-x\right| \leq n_{j}^{-5 / 12}} p_{n_{j}, s}(x)\left|\frac{k}{n_{i}}-\frac{s}{n_{j}}\right|=\frac{\sqrt{2 x(1-x)}}{\pi} \int_{-\infty}^{+\infty} \mathrm{d} w_{1} \int_{-\infty}^{+\infty}\left|\frac{w_{1}}{\sqrt{n_{i}}}-\frac{w_{2}}{\sqrt{n_{j}}}\right| \cdot \exp \left\{-w_{1}^{2}-w_{2}^{2}\right\} \mathrm{d} w_{2}+\circ\left(n^{-\frac{1}{2}}\right) \\
& =\frac{\sqrt{2 x(1-x)}}{\pi} \int_{-\infty}^{+\infty} \mathrm{d} w_{1} \int_{-\infty}^{+\infty}\left(\frac{w_{1}}{\sqrt{n_{i}}}-\frac{w_{2}}{\sqrt{n_{j}}}\right) \cdot \exp \left\{-w_{1}^{2}-w_{2}^{2}\right\} \mathrm{d} w_{2}+2 \frac{\sqrt{2 x(1-x)}}{\pi} \cdot \int_{-\infty}^{+\infty} \mathrm{d} w_{1} \int_{\sqrt{\frac{n_{j}}{n_{i}}}}^{+\infty}\left(\frac{w_{2}}{\sqrt{n_{j}}}-\frac{w_{1}}{\sqrt{n_{i}}}\right) \\
& \cdot \exp \left\{-w_{1}^{2}-w_{2}^{2}\right\} d w_{2}+o\left(n^{-\frac{1}{2}}\right) \\
& =\frac{\sqrt{2 x(1-x)}}{\pi} \int_{-\infty}^{+\infty} \frac{\mathrm{e}^{-\left(1+\frac{n_{j}}{n_{i}}\right) w_{1}^{2}}}{\sqrt{n_{j}}} \mathrm{~d} w_{1}+\frac{\sqrt{2 x(1-x)}}{\pi} \int_{-\infty}^{+\infty} \frac{\mathrm{e}^{-\left(1+\frac{n_{i}}{n_{j}}\right) w_{2}^{2}}}{\sqrt{n_{i}}} \mathrm{~d} w_{2}+\circ\left(n^{-\frac{1}{2}}\right) \\
& =\frac{\sqrt{2 x(1-x)}}{\pi}\left(\frac{1}{\sqrt{n_{j}}} \sqrt{\frac{n_{i} \pi}{n_{i}+n_{j}}}+\frac{1}{\sqrt{n_{i}}} \sqrt{\frac{n_{j} \pi}{n_{i}+n_{j}}}\right)+\circ\left(n^{-\frac{1}{2}}\right)=\frac{\sqrt{2 x(1-x)}}{\sqrt{\pi\left(n_{i}+n_{j}\right)}}\left(\sqrt{\frac{n_{i}}{n_{j}}}+\sqrt{\frac{n_{j}}{n_{i}}}\right)+\circ\left(n^{-\frac{1}{2}}\right) .
\end{aligned}
$$

Combining (11) and (20), we obtain

$$
\begin{aligned}
A_{3}(x) & =x-\frac{1}{2} \sum_{i=0}^{m-1} C_{i}(n) \sum_{j=0}^{m-1} C_{j}(n) \cdot \sum_{k=0}^{n_{i}} p_{n_{i}, k}(x) \sum_{s=0}^{n_{j}} p_{n_{j}, s}(x)\left|\frac{k}{n_{i}}-\frac{s}{n_{j}}\right| \\
& =x-\sqrt{\frac{2 x(1-x)}{2 \pi}} \sum_{i=0}^{m-1} C_{i}(n) \sum_{j=0}^{m-1} C_{j}(n) \cdot \frac{\sqrt{n_{i} / n_{j}}+\sqrt{n_{j} / n_{i}}}{\sqrt{n_{i}+n_{j}}}+\circ\left(n^{-\frac{1}{2}}\right) .
\end{aligned}
$$

From (5)-(7), (10), and (21), we complete the proof of Theorem 1.

\section{REFERENCES}

[1] K. Ritter, "Average-Case Analysis of Numerical Problems,” Springer-Verlag, Berlin, 2000.

[2] P. L. Butzer, "Linear Combinations of Bernstein Polynomials,” Canadian Journal of Mathematics, Vol. 5, 1953, pp. 559-567. http://dx.doi.org/10.4153/CJM-1953-063-7

[3] Z. Ditzian and V. Totik, "Moduli of Smoothness," Sprin-
ger-Verlag, Berlin, 1987. http://dx.doi.org/10.1007/978-1-4612-4778-4

[4] G. Q. Xu, "The Simultaneous Approximation Average Errors for Bernstein Operators on the R-Fold Integrated Wiener Space,” Numerical Mathematics Theory Methods and Applications, Vol. 5, No. 3, 2012, pp. 403-422.

[5] G. G. Lornetz, "Bernstein Polynomials," University of Toronto, Toronto, 1953. 